
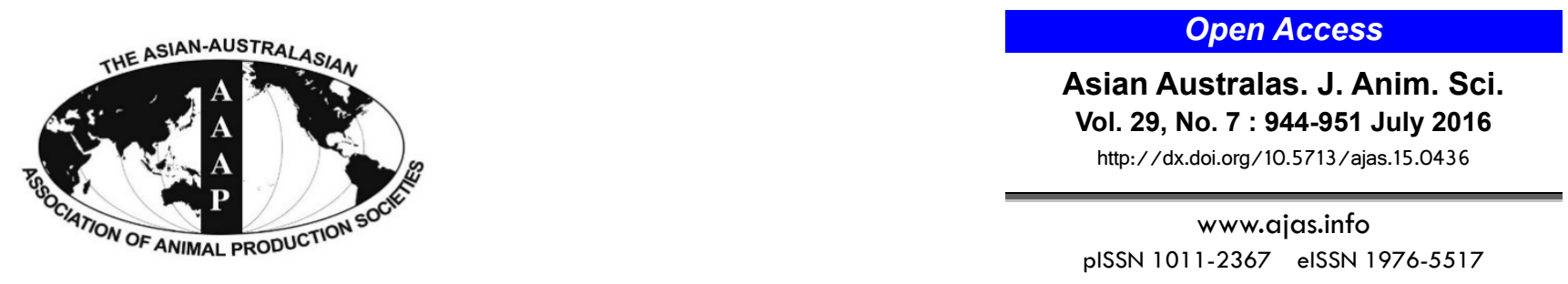

\title{
Tudor Domain Containing Protein TDRD12 Expresses at the Acrosome of Spermatids in Mouse Testis
}

\author{
Min Kima, Byeong Seong Kiª, Kwonho Hong', Se-pill Park², Jung-Jae Ko*, and Youngsok Choi* \\ Department of Biomedical Science, CHA University, 335 Pangyo-ro, Seongnam-si, Gyeonggi-do 13488, Korea
}

\begin{abstract}
T d r d 12$ is one of tudor domain containing ( $T d r d$ ) family members. However, the expression pattern of $T d r d 12$ has not been well studied. To compare the expression levels of $T d r d 12$ in various tissues, real time-polymerase chain reaction was performed using total RNAs from liver, small intestine, heart, brain, kidney, lung, spleen, stomach, uterus, ovary, and testis. Tdrd12 mRNA was highly expressed in testis. Antibody against mouse TDRD12 were generated using amino acid residues SQRPNEKPLRLTEKKDC of TDRD12 to investigate TDRD12 localization in testis. Immunostaining assay shows that TDRD12 is mainly localized at the spermatid in the seminiferous tubules of adult testes. During postnatal development, TDRD12 is differentially expressed. TDRD12 was detected in early spermatocytes at 2 weeks and TDRD12 was localized at acrosome of the round spermatids. TDRD12 expression was not colocalized with TDRD1 which is an important component of piRNA pathway in germ cells. Our results indicate that TDRD12 may play an important role in spermatids and function as a regulator of spermatogenesis in dependent of TDRD1. (Key Words: Tdrd12, Tudor, Spermatogenesis, Acrosome, Spermatid, Testis)
\end{abstract}

\section{INTRODUCTION}

Spermatogenesis is a complicated process in the testis. Once arrived at primitive embryonic testis, primordial germ cells are differentiated to spermatogonia. The sperm is differentially generated from spermatogonia via a series of meiotic process. At puberty, some of spermatogonia become primary spermatocytes entering meiosis and produce two secondary spermatocytes. Secondary spermatocytes turn to four haploid round spermatids by completion of meiosis. The round spermatids are transformed into sperms under

\footnotetext{
* Corresponding Authors: Jung-Jae Ko. Tel: +82-31-881-7132, Fax: +82-31-881-7249, E-mail: highko@cha.ac.kr / Youngsok Choi. Tel: +82-31-881-7149, Fax: +82-31-881-7249, E-mail: youngsokchoi@cha.ac.kr

${ }^{1}$ Department of Nanobiomedical Science \& BK21 PLUS NBM Global Research Center for Regenerative Medicine, Dankook University, Cheonan 330-714, Korea.

2 Department of Biotechnology, College of Applied Life Science, Jeju National University, Jeju 690-756, Korea.

a These authors are equally contributed.

Submitted May 16, 2015; Revised Aug. 31, 2015; Accepted Sept. 26, 2015
}

spermiogenesis (Matzuk and Lamb, 2008). The round spermatid has a unique cellular structure, a chromatoidbody in the cytoplasm (Meikar et al., 2011). The chromatoid-body contains a variety of RNAs and RNAbinding proteins that are involved in RNA protecting, decay, and silencing (Kotaja and Sassone-Corsi, 2007). Among RNA-processing proteins, tudor domain containing (TDRD) family proteins are important for germ-cell specific small RNA, piRNA biogenesis (Bak et al., 2011).

In mammals, 12 members of TDRD family proteins were identified since Drosophila tudor protein was discovered (Ying and Chen, 2012). Many members of TDRD family proteins are involved in germ cell development. For example, TDRD1 known as mouse tudor repeat-1 was originally found in spermatogonia and functions as an essential regulator for male germ-cell development (Wang et al., 2001; Chuma et al., 2003; Chuma et al., 2006). TDRD1 is highly expressed in fetal prospermatogonia and postnatal primary spermatocytes (Chuma et al., 2003). The localization of TDRD1 is exclusively restricted to the chromatoid bodies of late stage spermatocytes and round spermatids. $T d r d l$ deficient mice 
were sterile due to prevention of the meiotic process in the spermatocyte (Chuma et al., 2006). Tdrd2/Tdrkh was identified as a component in the Miwi complex (Chen et al., 2009). TDRD2 is critical for piRNA biogenesis in the germline with Miwi protein. Tdrd2 knockout mice are sterile resulting from the defect of spermatogenesis (Saxe et al., 2013). TDRD4/ring finger protein 17 (RNF17) and TDRD5 are mainly expressed in chromatoid bodies and involved in RNA processing for spermatogenesis (Smith et al., 2004; Pan et al., 2005). Tdrd5 deficiency in mice leads to spermatogenetic arrest at the round spermatid stage through unregulated retrotransposon silencing (Yabuta et al., 2011). TDRD6 is a regulator for miRNA function and plays an important role in chromatoid body organization and spermiogenesis (Vasileva et al., 2009). TDRD7 is ubiquitously expressed (Lachke et al., 2011; Tanaka et al., 2011). Tdrd7 disruption causes male sterility, cataract and glaucoma (Lachke et al., 2011; Tanaka et al., 2011). In the testis, TDRD7 is involved in suppression of long interspersed nuclear elements-1 (LINE-1) retrotransposons (Tanaka et al., 2011). TDRD8/Serine/Threonine kinase 31 (STK31) is expressed in mid-to-late spermatocyte cytoplasm and interacts with Piwi-like RNA-mediated gene silencing 1 (PIWI1) protein (Bao et al., 2012). TDRD9 also forms a protein complex with MIWI2 (Shoji et al., 2009). TDRD9 contains an ATPase/DExH-box ATPase (DExH)type helicase and a Tudor domain and functions in silencing LINE-1 retrotransposon during spermatogenesis. Deficiency of $T d r d 9$ in male mice is associated with sterility by failure of chromosome synapsis (Shoji et al., 2009). TDRD11/stapihylococcal nuclease domain containing 1 (SND1) is involved in multiple cellular process such as double-stranded RNA editing, pre-mRNA splicing, microRNA-mediating gene silencing and piRNA biogenesis in germlines (Callebaut and Mornon, 1997; Leverson et al., 1998; Yang et al., 2002; Caudy et al., 2003; Li et al., 2008; Paukku et al., 2008; Gao et al., 2012; Garcia-Lopez et al., 2013).

TDRD12 has been recently characterized as a TDRD family protein which has two tudor domains and a DEAD box. Drosophila TDRD12 ortholog is known to interact with the essential piRNA pathway factor Vreteno and regulates piRNA biogenesis in ovarian germline cells (Handler et al., 2011). TDRD12 in mice was also identified as a component of Piwi protein Piwi-like RNA-mediated gene silencing 2 (PIWI2) ribonucleoprotein complex including small RNAs, MILI and TDRD1, although TDRD12 does not directly interact with MILI and TDRD1. Tdrd12 deficiency in male mice induces atrophied testes (Pandey et al., 2013). The defects in Tdrd12 null mice resulted from loss of MIWI2-bound piRNA that is important for overall piRNA biogenesis. In this study, we generated anti-mouse TDRD12 antibody to investigate the expression pattern of TDRD12 in the mouse testis and demonstrated the differentially expressing TDRD12 in testis during postnatal development.

\section{MATERIALS AND METHODS}

\section{Animals}

All mice experiments were performed on 2 to 8 weekold ICR mice provided by Orient Bio Company (Seongnam, Korea). Mice were housed under temperature and light controlled conditions with the lights on for 12 hours daily and given with a free access to food and water. Care of mice and experimental procedures were conducted with the Guide for the Care and Use of Laboratory Animals (No. IACUC140032). All experiments were approved by the Institutional Animal Care and Use Committee of $\mathrm{CHA}$ University. Mouse testis was frozen and stored at $-80^{\circ} \mathrm{C}$ to isolate RNA and protein extraction or fixed in $4 \%$ paraformaldehyde solution to make paraffin block.

\section{Real time-polymerase chain reaction analysis}

Total RNA from testis was isolated using Trizol, as per the manufacturers' instruction (Invitrogen, Carlsbad, CA, USA). Two micrograms of total RNA were converted to cDNA using TOPscript cDNA Synthesis kit (Enzynomics, Daejeon, Korea), followed by PCR amplification. The primers used for real time-polymerase chain (RT-PCR) were as follows: Tdrd1 (5'-AGCAGAGACTGCTGTTGCAC-3' and 5'-CACAGCGGAGAGGCTAAAAG-3'), Tdrd2 (5'AAGGACATGGCCACAGAAAC-3' and 5'-CGTAGGA GCACAGGGAAGAG-3'), Tdrd3 (5'-ACGCAGCAGTTT TACCAAC-3' and 5'-CCCCATTGCCTTGTGTAAAT-3'), $T d r d 4$ (5'-CCAAGCCATAAAGGTGCTCT-3' and 5'TTATCTGCAAGCCCCATTTC-3'), Tdrd5 (5'-GGCACA AAAGGACTGGTGTT-3' and 5'-CAGGTTCAGACTGGC TCCTC-3'), Tdrd6 (5'-TCCAAACAGTGGGAAAGGAC$3^{\prime}$ and 5'-GACAACTTCCAGGGGTCAAA-3'), Tdrd7 (5'AGTGCTGCTTGGCTGATCTT-3' and 5'-GTTTCCACA GGTCTGCGTTT-3'), Tdrd8 (5'-AGCAGCCAGAAGGTT TTTGA-3' and 5'-GCAAGGTGGCAATGAACTTT-3'), Tdrd9 (5'-CACCAGTGATGGAGCTGAG-3' and 5'-GTGC ATTCTCCTGCAACTGA-3'), Tdrd11 (5'-GAATACCTGA ATGCCCAGG-3' and 5'-TTCAATGCTAGCCACGACAG3'), Tdrd12 (5'-CACCAGTTGCAGAAGGTGA-3' and 5'CGCAAAGCCCTGTATTTCAT-3'), and Gapdh (5'-AGGT CGGTGTGAACGGATTT-3' and 5'-GTAGACCATGTAG TTGAGGTCA-3').

\footnotetext{
Antibody generation and Western blot analysis

Antibody against mouse TDRD12 was generated by immunizing rabbits with the keyhole-limpet hemocyaninconjugated synthetic peptide SQRPNEKPLPLTEKKDC
} 
that corresponded to amino acid residues 318-334 (Youngin Frontier, Seoul, Korea). The antibody was affinitypurified and the specificity of the antibody was validated by Western blotting and immunofluorescence staining. Protein extract from testis was loaded on sodium dodecyl sulfatepolyacrylamide gel electrophoresis (4\% to $20 \%$ gradient gel), transferred to nitrocellulose membrane and blocked with 5\% non-fat milk in tri-buffered saline (TBS) containing $0.1 \%$ Tween 20 (TBST). The membrane was incubated with anti-TDRD12 and anti- $\alpha$-tubulin antibodies diluted in TBST-5\% milk at $4{ }^{\circ} \mathrm{C}$ for overnight, followed by incubation with horse radish peroxidase (HRP)-conjugated secondary antibody for 1 hour at room temperature. The membrane was developed using ECL Western Blotting substrate kit (GenDEPOT, Barker, TX, USA).

\section{Immunofluorescence}

The deparaffinized tissue section was incubated with blocking buffer (4\% bovine serum albumin and 5\% normal IgG in phosphate-buffered saline (PBS) for 1 hour at room temperature and incubated with primary antibody in the blocking buffer for overnight at $4^{\circ} \mathrm{C}$. The antibodies used in this study were anti-TDRD12 (1:200), Lectin-peanut agglutinin (PNA) $(1: 2,000$, Molecular Probes, Eugene, OR, USA), TDRD1 (1:200, R\&D systems, Minneapolis, MN, USA), and DEAD-box helicase 4 (DDX4) (1:500, Abcam, Cambridge, UK) antibodies. After washing with PBS, the sections were incubated with the secondary antibodies $(1: 1,000)$ in blocking buffer for 2 hours at room temperature. The testis sections were washed with PBS and mounted. 4',6-diamidino-2-phenylindole (DAPI) was used for nuclear counter stating of testis section. Slides were viewed and imaged with a confocal microscope, LSM 510 Meta (Carl Zeiss Co., Hamburg, Germany) equipped with three lasers.

\section{RESULTS}

\section{Expression of $\boldsymbol{T d} \boldsymbol{d} \boldsymbol{d}$ family members in the tissues}

To examine the expression of Tdrd family members in tissues, RT-PCR analysis was performed using total RNAs from various tissues; liver, intestine, heart, brain, kidney, lung, brain, uterus, ovary, and testis (Figure 1). The expression patterns of twelve Tdrd genes are shown in Figure 1A. Tdrd3, Tdrd7, and Tdrd11 were expressed in all tissues tested. The expression of Tdrd2, Tdrd5, and Tdrd12 was detected in several tissues. On the other hand, Tdrd1, $T d r d 4, T d r d 6, T d r d 8$, and $T d r d 9$ were exclusively expressed in the testis and/or the ovary (Figure 1A). These are consistent with previous reports showing $T d r d 1$, Tdrd2, $T d r d 4, T d r d 5, T d r d 6, T d r d 8, T d r d 9$, and $T d r d 12$ in germline cells. Tdrd12 was highly detected in the testis, whereas other tissues including the ovary and heart showed very low expression (Figure 1A). Next, we looked into the

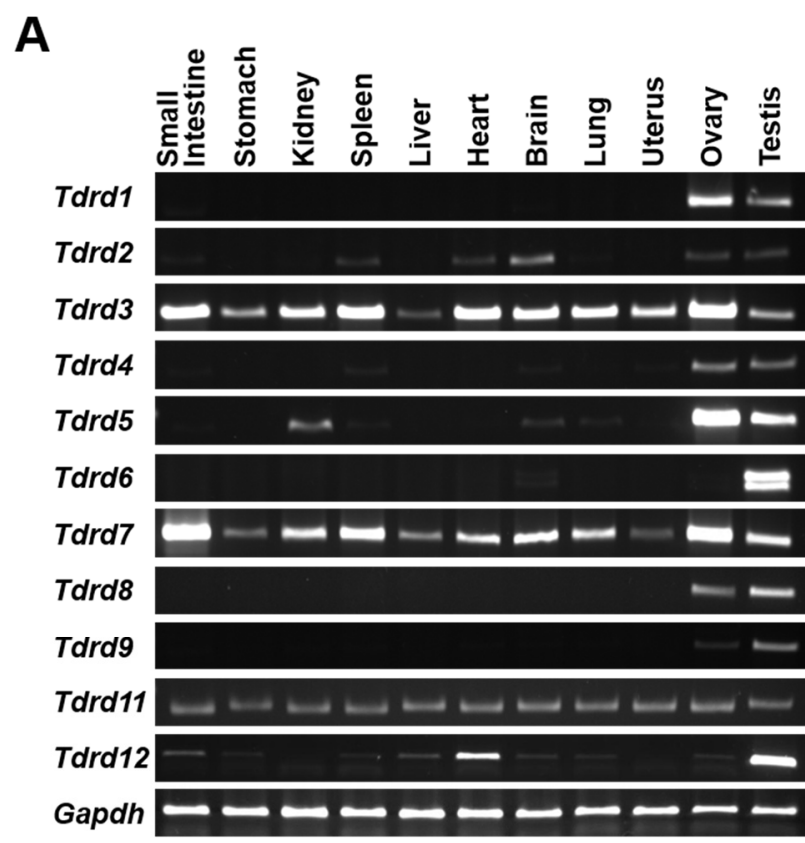

B

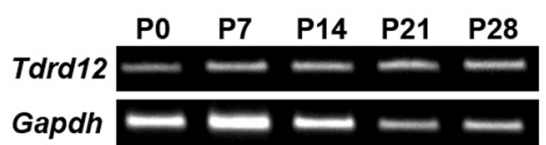

C

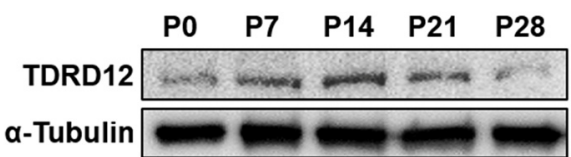

Figure 1. Gene expression of Tdrd family members in mouse tissues. (A) Expression of Tdrd family members were analyzed by RT-PCR. Total RNAs were extracted from mouse tissues; small intestine, stomach, kidney, spleen, liver, heart, brain, lung, uterus, ovary and testis. Gapdh was used as control. (B) Differential expression of Tdrd12 mRNA in the testis during postnatal development. Total RNAs were prepared from testes of postnatal day 0 (P0), P7, P14, P21, and P28 ICR mice. Gapdh was used as control. (C) Differential expression of TDRD12 in the testis during postnatal development. Testicular extracts were prepared from testes of postnatal day 0 (P0), P7, P14, P21, and P28 ICR mice. The alpha-tubulin ( $\alpha$-tubulin) used as loading control. Tdrd, tudor domain containing; RT-PCR, reverse transcriptionpolymerase chain reaction; Gapdh, glyceraldehyde 3-phosphate dehydrogenase; ICR, Institute for cancer research mice.

expression level of Tdrd12 mRNA in the testis during postnatal development (Figure 1B). Tdrd12 mRNAs were detected in the testis from postnatal day $0(\mathrm{P} 0)$ and then remained high to $\mathrm{P} 28$.

To analyze TDRD12 protein level in testis, we generated specific antibody against mouse TDRD12 using a small peptide SQRPNEKPLPLTEKKDC $\left(318^{\text {th }}\right.$ to 334 th amino acids of mouse TDRD12). TDRD12 ( 130 kDa) was detected in the testis during postnatal development at P0, P7, 
$\mathrm{P} 14, \mathrm{P} 21$, and $\mathrm{P} 28$. The protein was highly detected at P14 and then decreased at P21 (Figure 1C).

\section{Expression and localization of Tdrd12 protein in the testis}

To investigate the localization of TDRD12 in mouse testis, immunofluorescence was performed using antiTDRD12 antibody together with the stage specific markers. Anti-DDX4 antibody for germ cell, anti-TDRD1 antibody for spermatocyte, and anti-lectin-PNA antibody for spermatid marker were used for the stage specific markers in the testis. DDX4 was detected in the cytoplasm of spermatocyte and chromatoid body of round spermatid (Figure 2A). However, TDRD12 was not co-localized with DDX4. The staining pattern of TDRD12 was similar to a protein in acrosome structure in round spermatids (Figure 2A). To test whether TDRD12 is expressed in acrosome, we co-stained tissues with antibodies against TDRD12 and
lectin-PNA (Kallajoki et al., 1986). The localization of TDRD12 was completely overlapped with lectin-PNA in acrosome of round spermatid (Figure 2B). Next, we examined the co-localization of TDRD12 with TDRD1 which is a well characterized TDRD family member. TDRD12 was not co-localized with TDRD1 in the seminiferous tubule of testis, which was detected only in the spermatocyte, not in spermatid (Figure 3). This result suggested that the function of TDRD12 might not be associated with TDRD1 which acts as a molecular scaffold for piRNA biogenesis factors.

We also investigated the expression and localization TDRD12 in mouse testis during postnatal development at 2, 4,6 , and 8 weeks. At early stage of 2 weeks, TDRD12 was detected in the cytoplasm of primary spermatocyte (Figure 4). However, the expression of TDRD12 protein was localized in the acrosome of the spermatid at 4 week when the round spermatids are present in the seminiferous tubule.

A
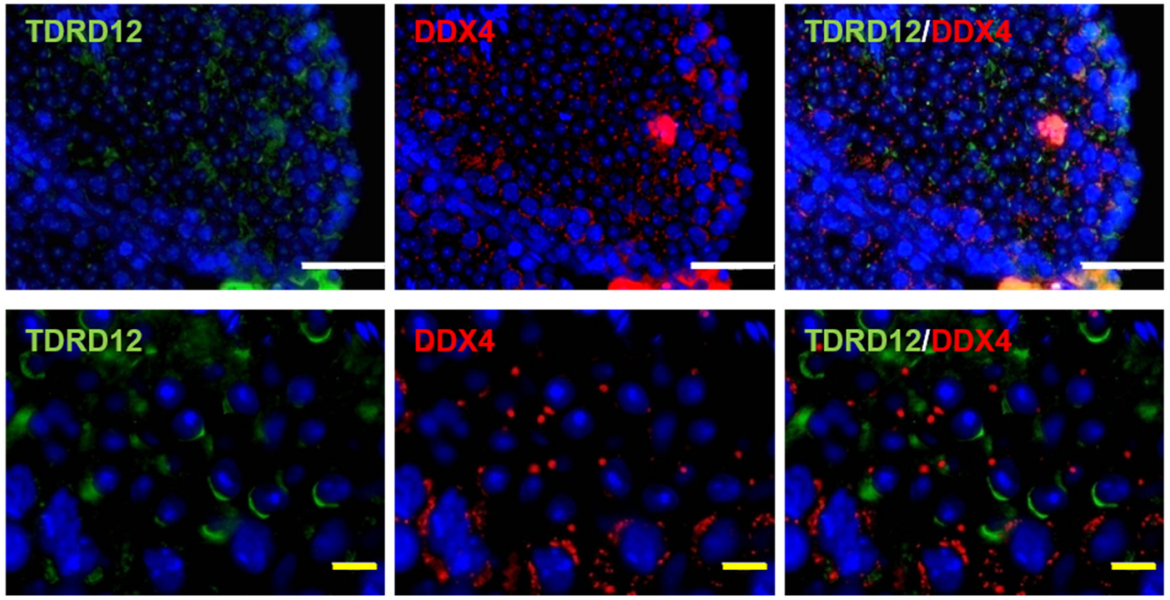

B
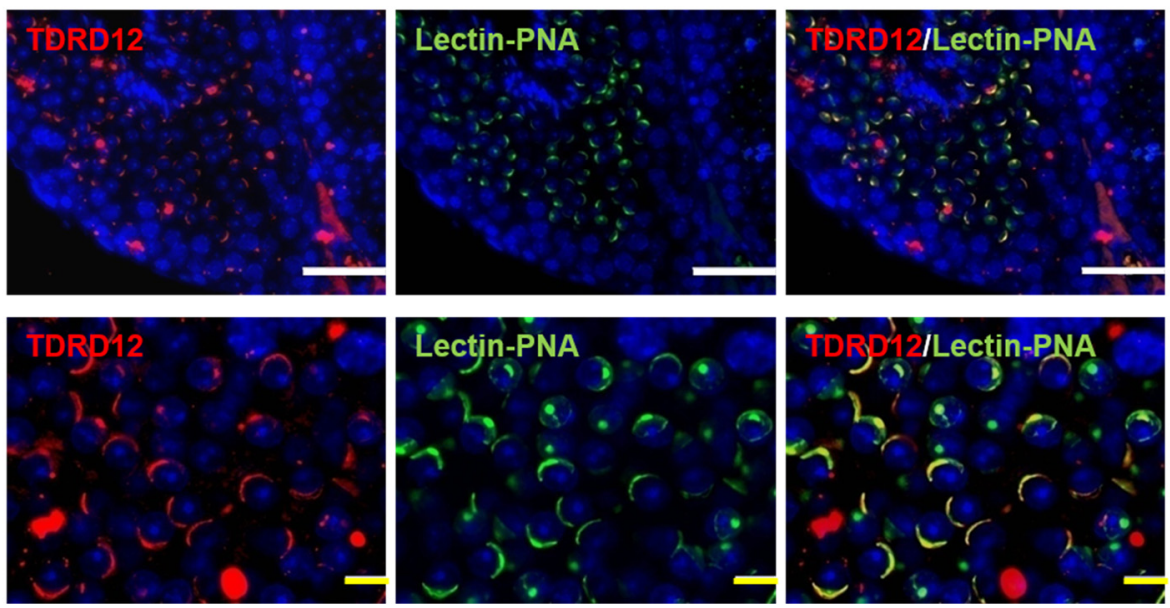

Figure 2. Localization of TDRD12 in acrosome of round spermatids. (A) Co-immunostaining of testis with antibodies against DDX4 and TDRD12. (B) Co-immunostaining of testis with antibodies against lectin-PNA and TDRD12. Tissue sections were prepared from 6weeks-old testis. White scale bars indicate $100 \mu \mathrm{m}$ and yellow bars indicate $10 \mu \mathrm{m}$. TDRD, tudor domain containing; DDX, DEAD-box helicase 4; PNA, peanut agglutinin. 

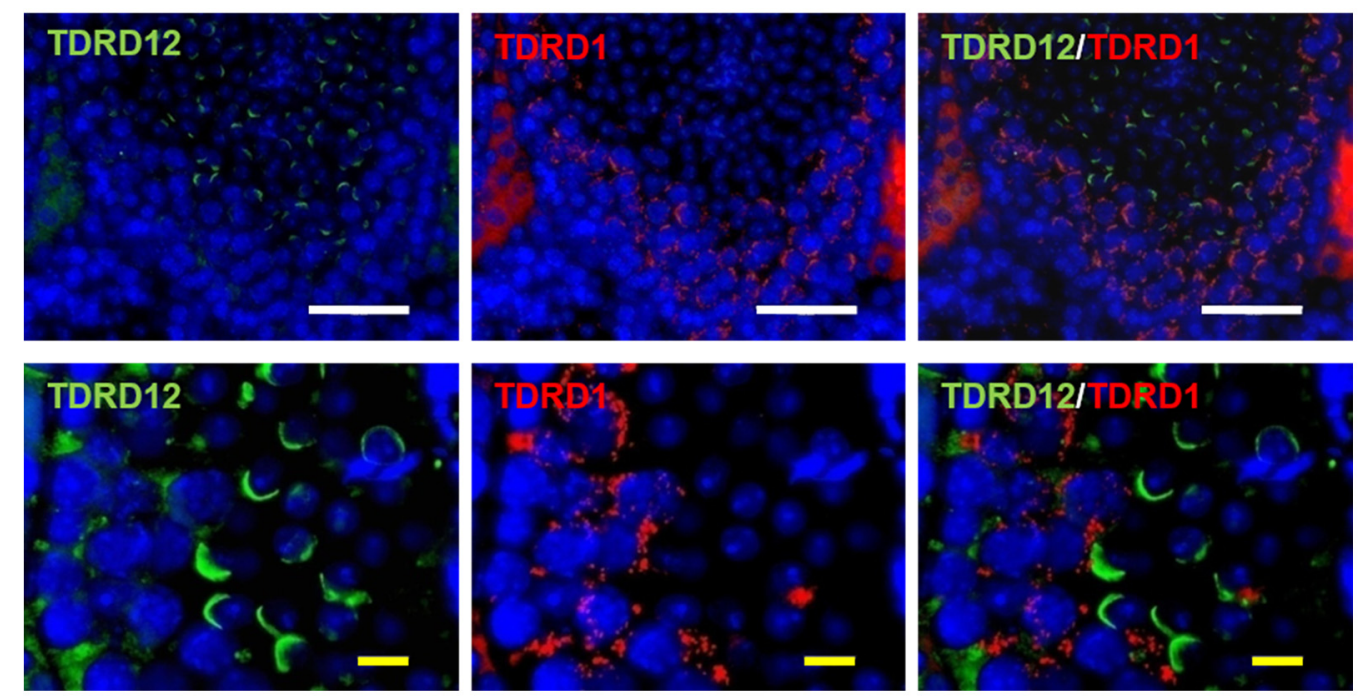

Figure 3. Co-localization of TDRD12 and TDRD1 in the mouse testis. Tissue sections were prepared from 6-weeks-old mouse. AntiTDRD12 and anti-TDRD1 antibodies were used for immunofluorescence. White scale bars indicate $100 \mu \mathrm{m}$ and yellow bars indicate 10 $\mu \mathrm{m}$. TDRD, tudor domain containing.

The localization of TDRD 12 was limited in the spermatids at 6 and 8 week (Figure 4).

\section{DISSCUSSION}

In this study we demonstrated that the expression of TDRD12 is limited at the acrosome of spermatids in the mouse testis. As described in the introduction, Tdrd family possesses 12 members including TDRD12. Their expressions vary in the tissues. $T d r d 3, T d r d 7$, and $T d r d 11$ ubiquitously express in various tissues. However, Tdrd1, $T d r d 5, T d r d 6, T d r d 8$, and $T d r d 9$ are exclusively expressed in the gonad, ovary and testis. This implies that they play an important role in the gonad. Mutant mice models show that these members regulate the development and differentiation of male gonad. The deficiency of Tdrdl causes the defect in the spermatogenesis and germinal granule formation in mice resulting in infertility of male mice (Chuma et al.,
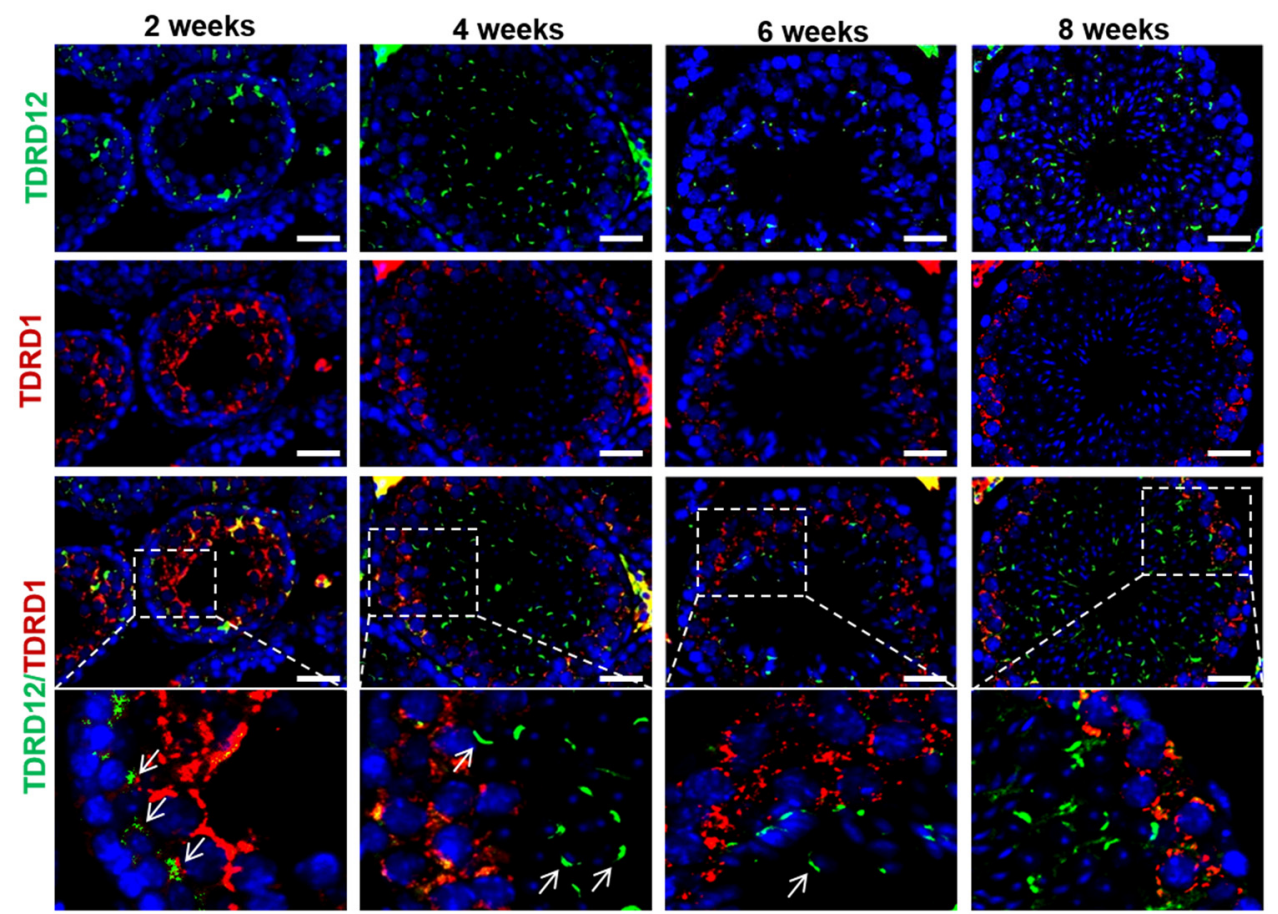

Figure 4. Differential expression of TDRD1 and TDRD12 in mouse testis during development. Tissue sections were prepared from 2, 4, 6 , and 8 weeks-old testis. Anti-TDRD12 and anti-TDRD1 antibodies were used for immunofluorescence. Arrow represents acrosome. Scale bars indicate $50 \mu \mathrm{m}$. TDRD, tudor domain containing. 
2006). Tdrd5 is essential for transposon silencing and spermiogenesis in the mice (Yabuta et al., 2011). Tdrd6 is also crucial for spermiogenesis by regulation of miRNA (Vasileva et al., 2009). The deficiency of $T d r d 7$ causes male sterility, age-related cataract and glaucoma (Lachke et al., 2011; Tanaka et al., 2011). In the testis, $T d r d 7$ is crucial for spermatid development by remodeling of chromatoid bodies (Tanaka et al., 2011). The disruption of Tdrd9 leads to the activation and increase of Line-1 transposons in the germ cell resulting in male infertility (Shoji et al., 2009). The TDRD9-MIWI2 complex cooperates with another tudor-piwi complex, Tdrd1-MILI, for the regulation of piRNA biogenesis and pathway. TDRD7 is also involved in regulation of retrotransposons independently of piRNA biogenesis.

The regulation of piRNA biogenesis is very important in germ cells. The piRNAs are germ-cell specific small noncoding RNAs and act through Piwi-like proteins (Piwil) and Tudor-domain repeat proteins (Bak et al., 2011). Piwil proteins include three family members; Piwill (MIWI1), Piwil2 (MILI), Piwil4 (MIWI2) (Bak et al., 2011). Piwil proteins interact with piRNA to form Piwil-piRNA complex which is critical for preventing desperate activity of transposable elements (Bortvin, 2013; Luteijn and Ketting, 2013). TDRD1 is very important for piRNA pathway during spermatogenesis. TDRD1 interacts with Piwil2 in the intermitochondrial cement (known as pi-body) (Aravin et al., 2009; Reuter et al., 2009). In the pi-body, the complex interacts with mouse vasa homolog Mvh RNA helicase (DDX4). Whereas, TDRD9-MIWIcomplex is present in piP-bodies (van der Heijden et al., 2010). The piP-bodies contain also DDX4. DDX4 plays a role for RNA substrate exchange between pi-bodies and piP-bodies as predicted by the ping-pong mechanism (Reynolds et al., 2005).

TDRD12 was not co-localized with either DDX4 or TDRD1 during spermatogenesis. TDRD12 is exclusively localized at the acrosome of the spermatid. This pattern is totally different from other members that are present in pibody or piP-body. The transcript of Tdrd12 seems to be hardly detected in several tissues. However, the expression of Tdrd12 was high in the testis and the heart compared to that in other tissues (Figure 1A). This implies that TDRD12 might play a role in the testis and the heart. Recent study revealed that $T d r d 12$ disruption causes male sterility and is very important for secondary piRNA biogenesis in mice (Pandey et al., 2013). However, the localization and expression pattern of TDRD12 protein remains unknown in the testis. We showed that Tdrd 12 was exclusively localized in the acrosome of spermatid. These results suggest that Tdrd12 might play an important role in spermatogenesis in the testis.

In this study, we showed that TDRD12 was exclusively localized in the acrosome of spermatid during spermatogenesis in the testis. It needs further investigation for a better understanding a role of TDRD12 in the acrosome of spermatid, which will provide a new function of tudor containing protein during germ cell development.

\section{AUTHOR CONTRIBUTIONS}

Experiment and writing (MK, BSK), Experiment (KH, $\mathrm{SP})$, and Experimental Design and writing (JJK, YC).

\section{CONFLICT OF INTEREST}

We certify that there is no conflict of interest with any financial organization regarding the material discussed in the manuscript.

\section{ACKNOWLEDGMENTS}

This research was supported by Basic Science Research and Priority Research Centers Program through the National Research Foundation of Korea (NRF) funded by the Ministry of Education (20090093821) and by grants from The Ministry for Food, Agriculture, Forestry and Fisheries, Cooperative Research Program for Agriculture Science \& Technology Development (PJ009103), Rural Development Administration, Republic of Korea.

\section{REFERENCES}

Aravin, A. A., G. W. van der Heijden, J. Castaneda, V. V. Vagin, G. J. Hannon, and A. Bortvin. 2009. Cytoplasmic compartmentalization of the fetal piRNA pathway in mice. PLoS Genet. 5:e1000764.

Bak, C. W., T. K. Yoon, and Y. Choi. 2011. Functions of PIWI proteins in spermatogenesis. Clin. Exp. Reprod. Med. 38:61-67.

Bao, J., L. Wang, J. Lei, Y. Hu, Y. Liu, H. Shen, W. Yan, and C. Xu. 2012. STK31(TDRD8) is dynamically regulated throughout mouse spermatogenesis and interacts with MIWI protein. Histochem. Cell Biol. 137:377-389.

Bortvin, A. 2013. PIWI-interacting RNAs (piRNAs) - a mouse testis perspective. Biochemistry (Mosc) 78:592-602.

Callebaut, I. and J. P. Mornon. 1997. The human EBNA-2 coactivator p100: multidomain organization and relationship to the staphylococcal nuclease fold and to the tudor protein involved in Drosophila melanogaster development. Biochem. J. 321:125-132.

Caudy, A. A., R. F. Ketting, S. M. Hammond, A. M. Denli, A. M. Bathoorn, B. B. Tops, J. M. Silva, M. M. Myers, G. J. Hannon, and R. H. Plasterk. 2003. A micrococcal nuclease homologue in RNAi effector complexes. Nature 425:411-414.

Chen, C., J. Jin, D. A. James, M. A. Adams-Cioaba, J. G. Park, Y. Guo, E. Tenaglia, C. Xu, G. Gish, J. Min, and T. Pawson. 2009 Mouse Piwi interactome identifies binding mechanism of Tdrkh Tudor domain to arginine methylated Miwi. Proc. Natl. 
Acad. Sci. USA 106:20336-20341.

Chuma, S., M. Hiyoshi, A. Yamamoto, M. Hosokawa, K. Takamune, and N. Nakatsuji. 2003. Mouse Tudor Repeat-1 (MTR-1) is a novel component of chromatoid bodies/nuages in male germ cells and forms a complex with snRNPs. Mech. Dev. 120:979-990.

Chuma, S., M. Hosokawa, K. Kitamura, S. Kasai, M. Fujioka, M. Hiyoshi, K. Takamune, T. Noce, and N. Nakatsuji. 2006. Tdrd1/Mtr-1, a tudor-related gene, is essential for male germcell differentiation and nuage/germinal granule formation in mice. Proc. Natl. Acad. Sci. USA 103:15894-15899.

Gao, X., X. Zhao, Y. Zhu, J. He, J. Shao, C. Su, Y. Zhang, W. Zhang, J. Saarikettu, O. Silvennoinen, Z. Yao, and J. Yang. 2012. Tudor staphylococcal nuclease (Tudor-SN) participates in small ribonucleoprotein (snRNP) assembly via interacting with symmetrically dimethylated Sm proteins. J. Biol. Chem. 287:18130-18141.

Garcia-Lopez, J., D. Hourcade Jde, and J. Del Mazo. 2013. Reprogramming of microRNAs by adenosine-to-inosine editing and the selective elimination of edited microRNA precursors in mouse oocytes and preimplantation embryos. Nucl. Acids Res. 41:5483-5493.

Handler, D., D. Olivieri, M. Novatchkova, F. S. Gruber, K. Meixner, K. Mechtler, A. Stark, R. Sachidanandam, and J. Brennecke. 2011. A systematic analysis of Drosophila TUDOR domain-containing proteins identifies Vreteno and the Tdrd12 family as essential primary piRNA pathway factors. EMBO J. 30:3977-3993.

Kallajoki, M., I. Virtanen, and J. Suominen. 1986. The fate of acrosomal staining during the acrosome reaction of human spermatozoa as revealed by a monoclonal antibody and PNAlectin. Int. J. Androl. 9:181-194.

Kotaja, N. and P. Sassone-Corsi. 2007. The chromatoid body: a germ-cell-specific RNA-processing centre. Nat. Rev. Mol. Cell Biol. 8:85-90.

Lachke, S. A., F. S. Alkuraya, S. C. Kneeland, T. Ohn, A. Aboukhalil, G. R. Howell, I. Saadi, R. Cavallesco, Y. Yue, A. C. Tsai, K. S. Nair, M. I. Cosma, R. S. Smith, E. Hodges, S. M. Alfadhli, A. Al-Hajeri, H. E. Shamseldin, A. Behbehani, G. J. Hannon, M. L. Bulyk, A. V. Drack, P. J. Anderson, S. W. John, and R. L. Maas. 2011. Mutations in the RNA granule component TDRD7 cause cataract and glaucoma. Science 331:1571-1576.

Leverson, J. D., P. J. Koskinen, F. C. Orrico, E. M. Rainio, K. J. Jalkanen, A. B. Dash, R. N. Eisenman, and S. A. Ness. 1998. Pim-1 kinase and p100 cooperate to enhance c-Myb activity. Mol. Cell 2:417-425.

Li, C. L., W. Z. Yang, Y. P. Chen, and H. S. Yuan. 2008. Structural and functional insights into human Tudor-SN, a key component linking RNA interference and editing. Nucl. Acids Res. 36:3579-3589.

Luteijn, M. J. and R. F. Ketting. 2013. PIWI-interacting RNAs: From generation to transgenerational epigenetics. Nat. Rev. Genet. 14:523-534.

Matzuk, M. M. and D. J. Lamb. 2008. The biology of infertility: Research advances and clinical challenges. Nat. Med. 14:11971213.

Meikar, O., M. Da Ros, H. Korhonen, and N. Kotaja. 2011.
Chromatoid body and small RNAs in male germ cells. Reproduction 142:195-209.

Pan, J., M. Goodheart, S. Chuma, N. Nakatsuji, D. C. Page, and P. J. Wang. 2005. RNF17, a component of the mammalian germ cell nuage, is essential for spermiogenesis. Development 132:4029-4039.

Pandey, R. R., Y. Tokuzawa, Z. Yang, E. Hayashi, T. Ichisaka, S. Kajita, Y. Asano, T. Kunieda, R. Sachidanandam, S. Chuma, S. Yamanaka, and R. S. Pillai. 2013. Tudor domain containing 12 (TDRD12) is essential for secondary PIWI interacting RNA biogenesis in mice. Proc. Natl. Acad. Sci. USA 110:1649216497.

Paukku, K., N. Kalkkinen, O. Silvennoinen, K. K. Kontula, and J. Y. Lehtonen. 2008. p100 increases AT1R expression through interaction with AT1R 3'-UTR. Nucl. Acids Res. 36:4474-4487.

Reuter, M., S. Chuma, T. Tanaka, T. Franz, A. Stark, and R. S. Pillai. 2009. Loss of the Mili-interacting Tudor domaincontaining protein-1 activates transposons and alters the Miliassociated small RNA profile. Nat. Struct. Mol. Biol. 16:639646.

Reynolds, N., B. Collier, K. Maratou, V. Bingham, R. M. Speed, M. Taggart, C. A. Semple, N. K. Gray, and H. J. Cooke. 2005. Dazl binds in vivo to specific transcripts and can regulate the pre-meiotic translation of Mvh in germ cells. Hum. Mol. Genet. 14:3899-3909.

Saxe, J. P., M. Chen, H. Zhao, and H. Lin. 2013. Tdrkh is essential for spermatogenesis and participates in primary piRNA biogenesis in the germline. EMBO J. 32:1869-1885.

Shoji, M., T. Tanaka, M. Hosokawa, M. Reuter, A. Stark, Y. Kato, G. Kondoh, K. Okawa, T. Chujo, T. Suzuki, K. Hata, S. L. Martin, T. Noce, S. Kuramochi-Miyagawa, T. Nakano, H. Sasaki, R. S. Pillai, N. Nakatsuji, and S. Chuma. 2009. The TDRD9-MIWI2 complex is essential for piRNA-mediated retrotransposon silencing in the mouse male germline. Dev. Cell 17:775-787.

Smith, J. M., J. Bowles, M. Wilson, R. D. Teasdale, and P. Koopman. 2004. Expression of the tudor-related gene Tdrd5 during development of the male germline in mice. Gene Expr. Patterns 4:701-705.

Tanaka, T., M. Hosokawa, V. V. Vagin, M. Reuter, E. Hayashi, A. L. Mochizuki, K. Kitamura, H. Yamanaka, G. Kondoh, K. Okawa, S. Kuramochi-Miyagawa, T. Nakano, R. Sachidanandam, G. J. Hannon, R. S. Pillai, N. Nakatsuji, and S. Chuma. 2011. Tudor domain containing 7 (Tdrd7) is essential for dynamic ribonucleoprotein (RNP) remodeling of chromatoid bodies during spermatogenesis. Proc. Natl. Acad. Sci. USA 108:10579-10584.

van der Heijden, G. W., J. Castaneda, and A. Bortvin. 2010. Bodies of evidence - compartmentalization of the piRNA pathway in mouse fetal prospermatogonia. Curr. Opin. Cell Biol. 22:752-757.

Vasileva, A., D. Tiedau, A. Firooznia, T. Muller-Reichert, and R. Jessberger. 2009. Tdrd6 is required for spermiogenesis, chromatoid body architecture, and regulation of miRNA expression. Curr. Biol. 19:630-639.

Wang, P. J., J. R. McCarrey, F. Yang, and D. C. Page. 2001. An abundance of X-linked genes expressed in spermatogonia. Nat. Genet. 27:422-426. 
Yabuta, Y., H. Ohta, T. Abe, K. Kurimoto, S. Chuma, and M. Yang, J., S. Aittomaki, M. Pesu, K. Carter, J. Saarinen, N. Saitou. 2011. TDRD5 is required for retrotransposon silencing, chromatoid body assembly, and spermiogenesis in mice. J. Kalkkinen, E. Kieff, and O. Silvennoinen. 2002. Identification of p100 as a coactivator for STAT6 that bridges STAT6 with Cell Biol. 192:781-795. RNA polymerase II. EMBO J. 21:4950-4958.

Ying, M. and D. Chen. 2012. Tudor domain-containing proteins of Drosophila melanogaster. Dev. Growth Differ. 54:32-43. 